Wan-Chen Lee. 2019. Cataloging practices through an ethnographic lens: workarounds, disagreements, and manifestations of culture. NASKO, Vol. 7. pp. 129-137.

Wan-Chen Lee - University of Washington

\title{
Cataloging practices through an ethnographic lens: workarounds, disagreements, and manifestations of culture
}

\begin{abstract}
Cataloging models emphasize selective aspects of cataloging and serve the purposes of conceptual debates and theoretical developments. Many complexities, uncertainties, dilemmas, challenges, and "rare" scenarios that catalogers encounter in practice are not presented in the models. To study cataloging practices, the author presents cataloging scenarios observed from an ongoing fieldwork. Through weekly participatory observations and unstructured interviews of catalogers, the work presents cases among the diverse and complex cataloging practices, and surfaces the tensions and time involved in cataloging. This paper will focus on three themes: workarounds, disagreements, and manifestations of culture in cataloging practice.

The first scenario describes a non-linear cataloging process and the different workarounds applied. The workarounds highlight the tacit knowledge of experienced catalogers. The second scenario shows catalogers' different perspectives about the authorship of stone rubbings. Disagreements, negotiation, and compromises in cataloging process are often not documented or explained. This scenario examines cataloging contexts that we cannot observe from analyzing cataloging standards or records. The third scenario describes the proposal of a Library of Congress Demographic Group Term (LCDGT): Zhiqing, and how it was approved as a LCSH: Zhiqing generation instead. The term encompasses a combination of regional, temporal, and cultural aspects of a demographic group. In the proposal process, I identified cultural manifestations in cataloging process through observing "the missing pieces" and local adaptations. This study contributes to the knowledge organization literature by presenting cataloging scenarios that require prolonged engagement to study.
\end{abstract}

\section{Introduction}

In the context of libraries, cataloging refers to the process by which resource descriptions are created. During the cataloging process, many critical decisions are made. The cataloging decisions identify and represent the relationships between resources, and shape users' interactions with the resources. In the knowledge organization literature, scholars have recognized the challenges and complexities of cataloging and its components, such as subject analysis and name authority control (Cheng 2018; Taylor \& Joudrey 2009). Scholars build models to examine specific aspects of the components of cataloging. Take subject analysis for example, Wilson (1968) proposes approaches for subject analysis, while Langridge (1989) and Taylor and Joudrey (2009) identify steps in subject analysis. Hjørland (2011) examines indexing, one other component of cataloging, and distinguishes indexing approaches by epistemological stances. These are among the many attempts to parse and model the cataloging process from different perspectives. These models emphasize particular aspects of cataloging at the conceptual level, and contribute to conceptual debates and theoretical developments. However, these models do not often present the rich and specific contexts and challenges in cataloging practices.

Models simplify complex things by providing general and typical categorizations or actions. They direct our attention to the criteria from which the models are built. Simplification and selective emphasis are the strengths of models that contribute to conceptual debates and theoretical developments. As a tradeoff, the depictions of the topic 
Wan-Chen Lee. 2019. Cataloging practices through an ethnographic lens: workarounds, disagreements, and manifestations of culture. NASKO, Vol. 7. pp. 129-137.

(e.g., cataloging) in models are the ideal or typical cases. Many complexities, uncertainties, and dilemmas in cataloging practice are not presented in the models.

Recognizing the gap between models and practice, we may ask: how does one study cataloging practices? Previous studies apply variant approaches. Porter and Bredderman (1997) use survey to understand the challenges faced by catalogers who describe non- print resources in academic libraries. Sauperl and Saye (1998) look into the process of subject cataloging by observing 5 catalogers. They apply a combination of observation, think-aloud method, and unstructured interview. Thompson (2016) examines how gender is described in name authority records through content analysis of the records. Silva, Ferreira, and Martins (2016) use a case study to present the challenges and achievements of adapting and developing local cataloging practice and rules for manuscripts. While these works help improve our understanding of cataloging practice, we still lack detailed accounts of complexities, uncertainties, dilemmas, challenges, and "rare" scenarios. Ethnographic studies on cataloging practice can help. In this paper, I present several cataloging scenarios from my ongoing fieldwork. In contrast to models, my ethnographic work surfaces the tensions and time involved in cataloging. I will focus on three: workarounds, disagreements, and manifestations of culture in cataloging practice.

\section{Methodological Context}

Since September 2015, I started an ethnographic study which explores the manifestations of culture in cataloging practice (Lee 2017). In this study, I shadow a cataloger Q (pseudonym) at a research I university library once a week. In the field, I conduct participatory observations and informal interviews and take field notes. Occasionally, there are opportunities to observe interactions between $Q$ and other librarians. One of the cataloging scenarios I present is the interaction between $\mathrm{Q}$ and one other cataloger $\mathrm{Z}$ (pseudonym). They both gave consent to publishing my observations in the field. Q is experienced in applying international and U.S. standards to catalog materials in different formats and languages. As a native Chinese speaker, Q not only catalogs English and Chinese materials, but also collaborates with other librarians to describe materials in Japanese, Korean, and some European languages. $\mathrm{Z}$ is a professor and a cataloger in China who specializes in Chinese rare books and special collections. He was invited as a subject expert to participate in a cataloging project for Chinese special collections. The fieldwork focuses mainly on one cataloger's work. However, through prolonged engagement and thick description, the ethnographic approach has generated more than 130 fieldnotes, which allow me to observe cataloging scenarios that span across a long period of time as well as draw out themes that surface from multiple scenarios.

\section{Scenarios}

This section will present three cataloging scenarios as examples of workarounds, disagreements, and manifestations of culture. The first scenario depicts how the cataloger Q tried different workarounds to complete the task of description. The second scenario has two parts. The first part shows the disagreement between $\mathrm{Q}$ and $\mathrm{Z}$ on the authorship of stone 
Wan-Chen Lee. 2019. Cataloging practices through an ethnographic lens: workarounds, disagreements, and manifestations of culture. NASKO, Vol. 7. pp. 129-137.

rubbings. The second part describes another disagreement between $\mathrm{Q}$ and a different librarian on the same topic, which reinforces the theme in the first part. The third scenario describes the proposal of a Library of Congress Demographic Group Term (LCDGT): Zhiqing, and how it was approved, with modifications, as a subject heading in LCSH: Zhiqing generation.

\section{Workarounds}

The cataloger's workarounds began with the search of author information of a reproduction of the manuscript -- fen xi "Zhou yi" shi dai zhi she hui bei jing (卦爻辞解释: 分析《周易 》时代之社会背景). The text presents analysis of the social context in the "Zhou yi" era (between the 10th and 4th centuries BCE). According to the copy in hand, the text was written by $\mathrm{Ce} \mathrm{Ni}$, who studied Yijing (i.e., the Book of Changes, a Chinese classic) and was affiliated with Cornell University. There was no name authority record for Ce Ni. As a component of creating a bibliographic record for the text, $\mathrm{Q}$ had to create a name authority record for $\mathrm{Ce}$ $\mathrm{Ni}$. To create one, Q examined the text, but found the author information in the text insufficient. Online searches did not provide relevant information about $\mathrm{Ce} \mathrm{Ni}$ either. There were only two leads left. First, Ce Ni's family donated his book collection to UC Irvine libraries. Second, on the cover, a line specifies that Bilun Shi, a professor in Cornell University, provided guidance and feedback to the author. While there was a LC name authority record for Bilun Shi (i.e., Shi, Bilun, 1931- ), Q could only guess that Bilun Shi and Ce Ni might be colleagues. Following the first lead, Q sent an email to UC Irvine libraries, asking for Ce Ni's information. The librarian who processed the donated collection left the position, but a librarian helped forwarding Q's inquiry to the librarian and the donor. With little hope, $\mathrm{Q}$ waited for responses from the far-stretched lead. The creation of Ce Ni's name authority record came to a halt.

Four months after the first attempt of cataloging the text, Q discussed this challenging case with a reference librarian, who found an obituary of Ce Ni's wife from the Cornell Chronicle. In the obituary, $\mathrm{Ce} \mathrm{Ni}$ was described as "Tseh $\mathrm{Ni}$, a librarian in Cornell's East Asian Collection from 1962 to 1986, died in 1997" (Cornell Chronicle 2016). The discovery was critical in that it highlighted the discrepancies caused by different Romanization standards. "Ce Ni" is the Romanized form using the Pinyin standard, and "Tseh Ni" is the form using Wade-Giles. Even after implementing the Library of Congress Pinyin Conversion Project (Library of Congress 2005), there are still traces of Wade-Giles and other Romanization standards in resources and resource descriptions. Using "Ce Ni" as the only query term missed relevant information. Using the new information about $\mathrm{Ce} \mathrm{Ni}$, the reference librarian searched "Tseh Ni" on BillionGraves.com, and found his birth and death dates. Q finally collected enough information and created the name authority record for $\mathrm{Ce} \mathrm{Ni}$.

\section{Disagreements}

Disagreements on the authorship of rubbings arise from discussions between $\mathrm{Q}$ and $\mathrm{Z}$, and again between $Q$ and another librarian in Taiwan. The first part of this scenario happened in a cataloging project for Chinese special collections. $Z$ used his expertise in Chinese special collections to examine and catalog rubbings. $Q$ reviewed the records to make sure they 
Wan-Chen Lee. 2019. Cataloging practices through an ethnographic lens: workarounds, disagreements, and manifestations of culture. NASKO, Vol. 7. pp. 129-137.

comply with RDA (Resource Description and Access) rules. RDA is based on the FRBR WEMI model, but it is challenging to use the model to describe a resource type like rubbing. A rubbing is created based on two Works: the original artwork (e.g., painting, calligraphy), and a stone stele. The "lifecycle" of the artwork starts with its creation on paper by artist(s) or calligrapher(s). To extend the longevity of the artwork, the owner may hire engraver(s) to carve a representation of the artwork in a stele. When the stele decays, people could further preserve the artwork by hiring technician(s) to create rubbings. Technicians attach a paper to the stele and pad ink against the paper to transfer the content of the stele to the paper which creates the rubbing. It is possible to create multiple steles of an artwork, or create multiple rubbings of a stele at different times. The engravers and technicians may also differ. Further, rubbings reflect the conditions of the stele, which may change over time.

To collaborate on cataloging rubbings, Q explained the FRBR WEMI model and its application to Z. Q treated the original artwork, the stele, and the rubbing as three related but distinct Works. These works have different creators (artist/calligrapher, engraver, and technician respectively) and creation dates. The cataloging record of a rubbing can note the relationships between the three works, but the creator of a rubbing should be the technician. However, $\mathrm{Z}$ viewed rubbing as reproduction of the original artwork. Oftentimes, the original artwork no longer exists, and the stele is preserved in a remote site. Rubbing may be the only accessible option for many users. $\mathrm{Z}$ argued that it is the original artwork that users care about. Therefore, to help users find rubbings, catalogers should record the creator of the original artwork as the creator of the rubbings. $\mathrm{Z}$ also provided some cultural context and explained that in pre-modern China, engravers and technicians were treated as mechanics. They were not creators of artworks. Hence, most of their names were not documented. From Z's perspective, if we describe rubbings using RDA and see rubbing as its own work, the author information (i.e., the technicians) and publication information would likely be "unknown." Cataloging records created like this would fail to serve the finding and selecting objectives of the catalog (Svenonius 2000). After several communications, Q and Z still disagree and felt frustrated. Q said, "there is a big gap, as great as the Grand Canyon, between the West and China." $\mathrm{Z}$ also told me that "the RDA-way of cataloging is flipping my records upside down."

Three years later, Q gave a talk at the National Central Library in Taiwan and shared her experiences cataloging rubbings. After the talk, a cataloger at the library disagreed with $\mathrm{Q}$ and had a debate with her about the authorship of rubbings. The cataloger agreed with $\mathrm{Z}$ and preferred recording the creators of the original artworks as the creators of rubbings. However, Q pointed out the fundamental differences between the three works to persuade the librarian. When an original artwork was engraved on a stone stele, it transformed from a 2D format to a 3D object through the hands of an engraver. Should we treat the artwork and the stele as the same work? Similarly, when a technician transfers the content on a stele to a rubbing, there is a transformation from a $3 \mathrm{D}$ object to a $2 \mathrm{D}$ format. Should we treat the stele and the rubbing as the same work? After discussions, the catalogers agreed that we need more studies on the authorship of rubbings. 
Wan-Chen Lee. 2019. Cataloging practices through an ethnographic lens: workarounds, disagreements, and manifestations of culture. NASKO, Vol. 7. pp. 129-137.

\section{Manifestations of Culture}

In this scenario, I observed the process of proposing a Library of Congress Demographic Group Term (LCDGT) -- Zhiqing, and identified manifestations of culture. According to Guo, Song, and Zhou (2006, 74), the educated youths (also known as zhishi qingnian or zhiqing) "refers mainly to urban and suburban middle-school and high- school graduates during the Cultural Revolution who were sent to the countryside to work, to settle down, and to be "reeducated" by the farmers there." The term covers a combination of geographical, temporal, and cultural aspects of a demographic group. However, it was not an authorized descriptor in LCSH or LCDGT. The cataloger Q looked up records of works about zhiqing or works written by zhiqing. The common description is a combination of these LCSHs: "Youth--China", "China--History-- Cultural Revolution, 1966-1976", and "Down to the countryside movement (China)". Many works on this topic were classed under DS778.7 in the Library of Congress Classification, which emphasizes the history of the Cultural Revolution. The demographic group was a poorly represented by the headings available.

The first LCDGT proposal for zhiqing was submitted in May, 2017. In August, 2017, the proposal was rejected (Library of Congress 2017), because (1) the meaning of this term changed and it was "not possible to establish an unambiguous heading for this class of persons, in either English or Chinese." Zhiqing was first used in the May Fourth movement in 1919. At the time, the term translated to "educated youth." In the 1950s to 1970s, zhiqing referred to sent-down youth in the Down to the countryside movement. Another reason of rejection was that (2) zhiqing can loosely translate to "educated youth." The heading "Down to countryside movement (China)" should be sufficient in describing works about zhiqing. After the decision was posted, Q collected comments from East Asian libraries in the U.S. She compiled a document to respond to the posted reasons of rejection, and demonstrated the necessity of establishing this heading. The document cites studies of zhiqing and dictionaries that define zhiqing. These resources show that zhiqing is the term widely used in scholarly publications, library collections, and conferences. While some scholars use "educated youth," the term is insufficient for capturing the characteristics of this demographic group. The document includes a case that shows how an East Asian library developed a local solution to add zhiqing as a searchable metadata, because users do not use "Youth--China" to search for resources about zhiqing. In the document, $\mathrm{Q}$ also points out that there are more than 17 million zhiqing (Guo, Song \& Zhou 2006, 268) and many are contemporary political leaders and influential writers. The document was sent to LC in March, 2018, requesting reconsideration of the LCDGT proposal for zhiqing.

In May, 2018, LC rejected the LCDGT proposal for zhiqing, but approved a different form: Zhiqing generation, as a LCSH. The term was added as a LCSH because proposals for LCDGT were no longer accepted since February, 2018 (Library of Congress 2018). LC explained the approval and modification of the heading. Q shared what she learned with me. According to the explanation, the second proposal (i.e., the document sent in March, 2018) includes more citations of quality sources, and it mentions that many contemporary political leaders are zhiqing. This is a strong piece of evidence that justifies zhiqing as a shared identity among a demographic group. The information contributed to the approval of the term. 
Wan-Chen Lee. 2019. Cataloging practices through an ethnographic lens: workarounds, disagreements, and manifestations of culture. NASKO, Vol. 7. pp. 129-137.

However, the authorized form of this heading is zhiqing generation. The form was selected because (1) using zhiqing alone is ambiguous, and (2) there are precedent headings such as Baby boom generation.

\section{Discussion}

The first scenario on workarounds is an example of a non-linear cataloging process. It shows how the go-to resources may fail the purpose of description. As a result, a cataloger needs to be creative and apply tacit knowledge to come up with workarounds. In the scenario, Q consulted multiple sources, and found critical information through unexpected routes. Through ethnographic study, I was able to document the details of several attempts to create and revise a name authority record which span across a 4- month period. This approach captures the behind-the-scene stories that may not contribute directly to the end product (i.e., bibliographic and authority records) but may shed light on cataloging practice.

The second scenario on disagreements highlights two themes: (1) rubbing as a complex resource type, and (2) conflicting semantic warrants. Rubbing is closely related to an artwork and a stele. In the scenario, $\mathrm{Z}$ used the information of the original artwork to describe a rubbing, while $Q$ focused on describing the rubbing at hand. The different focus of description is a major factor that leads to disagreements. The complexity of resource types can add to the complexity of descriptions. This scenario is also an example of conflicting semantic warrants in cataloging practices. Semantic warrants is an umbrella term for rationales which justify classificatory decisions, such as literary warrant, user warrant, and cultural warrant (Beghtol 1986; 2002; Kwasnik 2010). The semantic warrants underlying Q's approach include content warrant and standard warrant (the transcription principle, RDA, and WEMI model). The transcription principle has an assumption that cataloging records serve as faithful surrogates of the entity described. On the other hand, $\mathrm{Z}$ advocates for an approach that prioritizes user warrant and cultural warrant. The assumed user need is to access the original artwork or the most accessible representation of it. Z's argument about the roles of engravers and technicians was based on the cultural contexts in which the artworks, steles, and rubbings were created.

The third scenario allows us to identify manifestations of culture in cataloging practice. Zhiqing is a term with particular geographical, temporal, and cultural context. Through observing the motivation, communication, and challenges in the proposal process, some themes surface and provide examples of how culture manifests in knowledge organization. (1) Language is a prominent theme. It reinforces the motivation of the proposal -- to recapture what was lost in the unsatisfactory translation and description (e.g., educated youth and Youth--China). Language is also critical in determining the preferred form of a heading. Q explained that for foreign concepts, LC prefers using the English translation as the preferred term. When an English translation is not available, a partial translation is used. An example is the name authority file for Tainan, a city in Taiwan. While the Romanization of the city is Tainan Shi, the preferred form is Tainan City (Taiwan). In this case, the concept Shi is translated to City. If no English translation is available, a preferred term can be in Romanized form. For example, Yue fu, a genre of Chinese poems that are derived from folk songs, is a 
Wan-Chen Lee. 2019. Cataloging practices through an ethnographic lens: workarounds, disagreements, and manifestations of culture. NASKO, Vol. 7. pp. 129-137.

preferred term. In this scenario, language is closely related to the selection of preferred term and the motivation of proposing the heading. (2) Culture can manifest in ambiguity. Ambiguity is noted in both proposals, first as a reason of rejection, and then as a reason of modification. In the initial proposal, the two meanings of zhiqing contribute to its ambiguity. This is not a culturally specific challenge. In the second proposal, Q addressed this issue and suggested adding a heading for each meaning and disambiguate them. Q proposed a solution to use "zhishi qingnian (1919)," the full term of zhiqing with qualifier, for educated youth in the May Fourth Movement; and use the common abbreviation "zhiqing" for sent-down youth in the 1950s to 1970s. However, the solution was not accepted. Zhiqing generation became the preferred term. We can explore why one approach was preferred over another, and the assumptions and semantic warrants underlying each approach. Q's approach is based on literary warrant, scholarly warrant, and cultural warrant. Zhiqing is commonly used in scholarly publications and literature. In addition, applying date as qualifier helps present the cultural context of the heading. On the other hand, the LC's approach prioritizes standard warrant, user warrant, and cultural warrant. Selecting the English translation as preferred term not only follows the principle and takes precedent headings into account, but also reflects the target users. Through an ethnographic approach, we can analyze the rationales for term selection, and observe the prioritization of semantic warrants. (3) Culture can also manifest in the localizations of cataloging practice. The second proposal includes a case of an East Asian library. The library uses localized solution to make zhiqing searchable. Since localization often requires extra costs, it highlights the significant things that cannot be compromised. Manifestations of culture may share the uniqueness and fall within this category of things. The scenario demonstrates how researchers may identify cultural influences in cataloging through observing changes and adaptations using an ethnographic approach.

\section{Conclusion}

When viewed through conceptual models, the cataloging process may seem linear or uniformed. To harvest the richness of cataloging in practice, many research methods could be applied. This paper focuses on presenting three cataloging scenarios collected through ethnographic approaches. Prolonged engagement in the field allows researchers to parse the complexities in cataloging practice, and address topics that require in-depth analysis. The three cataloging scenarios outlined above are examples of such topics: workarounds, disagreements, and manifestations of culture.

Workarounds are often tacit knowledge of experienced catalogers. Ethnographic study is a good approach to identify what has been tried, what has failed, and how a cataloger created the latest description. Similarly, disagreements, negotiation, and compromises in cataloging process are often not documented or explained. This study looks into the behind-the-scene contexts to capture what we cannot observe from analyzing cataloging standards or records. By examining the episodes of trial and error and the Eureka moments in cataloging processes, we can advance our understanding of cataloging. For instance, we may draw attention to the challenges catalogers face, shed light on concerns in standards, or trace the rationale and 
Wan-Chen Lee. 2019. Cataloging practices through an ethnographic lens: workarounds, disagreements, and manifestations of culture. NASKO, Vol. 7. pp. 129-137.

semantic warrant embedded in cataloging records. In addition, the study identifies and describes manifestations of culture. Culture is a complex and ambiguous concept. While the concept of culture seems ubiquitous, there is no consensus on its definition. Previous studies in knowledge organization have discussed the ethical concerns and tension between standardization and localization for local cultures (Cheng 2018; Diao \& Cao 2016; Lee 2016; López- Huertas 2013; Olson 2002), and the importance of cultural warrant (Beghtol 1986; 2002). Building on these works, we look at where and how culture manifests in cataloging practices. Cultural manifestations were identified through observing "the missing pieces" and local adaptations in cataloging process. This study contributes to the knowledge organization literature by presenting cataloging scenarios on three topics that require prolonged engagement to study.

\section{References}

Beghtol, C. 1986. Semantic Validity: Concepts of Warrant in Bibliographic Classification Systems. Library Resources and Technical Services 30(2): 109-25.

Beghtol, C. 2002. A Proposed Ethical Warrant for Global Knowledge Representation and Organization Systems. Journal of Documentation 58(5): 507- 32.

Beghtol, C. 2005. Ethical Decision-making for Knowledge Representation and Organization Systems for Global Use. Journal of the American Society for Information Science and Technology 56(9): 903-12.

Cheng, H. 2018. Glocalization and Other Challenges to Cataloging Chinese Continuing Resources. Cataloging \& Classification Quarterly 56(2-3): 146-54.

Cornell Chronicle. 2016, October 16. Pei Shin Nei, Clark Award winner, dies at 86. Retrieved May 8, 2019, from Cornell Chronicle website: http://news.cornell.edu/stories/2006/10/pei-shin-nei-clarkaward-winner-dies-86

Diao, J. and H. Cao. 2016. Chronology in Cataloging Chinese Archaeological Reports: An Investigation of Cultural Bias in the Library of Congress Classification. Cataloging \& Classification Quarterly 54(4): 1-19.

Guo, J., Y. Song and Y. Zhou. 2006. Historical Dictionary of the Chinese Cultural Revolution. Lanham, Md.: Scarecrow Press.

Hjørland, B. 2011. The Importance of Theories of Knowledge: Indexing and Information Retrieval as an Example. Journal of the American Society for Information Science and Technology 62(1): 72-7.

Kwasnik, B. H. 2010. "Semantic Warrant: A Pivotal Concept for Our Field." Knowledge Organization 37(2): 106-10.

Langridge, D.W. 1989. Subject Analysis: Principles and Procedures. London; New York: BowkerSaur.

Lee, W. 2016. Challenges and Considerations of Adapting Foreign Classification Standards. In Knowledge Organization for a Sustainable World: Challenges and Perspectives for Cultural, Scientific, and Technological Sharing in a Connected Society: Proceedings of the Fourteenth International ISKO Conference 27-29 September 2016 Rio de Janeiro, Brazil, eds. José Augusto Chaves Guimarães, Oliveira Milani, S. Dodebei, V. Advances in knowledge organization 15. Würzburg: Ergon Verlag, 485-92.

Lee, W. 2017. Conflicts of Semantic Warrants in Cataloging Practices. In Proceedings from North American Symposium on Knowledge Organization 15-16 June 2017 Urbana-Champaign, Illinois. 231-8. 
Wan-Chen Lee. 2019. Cataloging practices through an ethnographic lens: workarounds, disagreements, and manifestations of culture. NASKO, Vol. 7. pp. 129-137.

Library of Congress. 2005. Library of Congress Pinyin Conversion Project. Retrieved May 8, 2019, from https://www.loc.gov/catdir/pinyin/

Library of Congress. Policy and Standards Division. 2017. Summary of Decisions, Editorial Meeting Number 08. Retrieved May 15, 2019, from Library of Congress website: http://www.loc.gov/aba/pcc/saco/cpsoed/psd-170821.html

Library of Congress. Policy and Standards Division. 2018. Phase 3 of Library of Congress Demographic Group Terms Development Ends. Retrieved May 15, 2019, from Library of Congress website: https://www.loc.gov/catdir/cpso/lcdgt- developmentend.html

López-Huertas, M.J. 2013. Transcultural Categorization in Contextualized Domains. Information Research 18(3): paper C16.

Olson, H. A. 2002. The Power to Name: Locating the Limits of Subject Representation in Libraries. Springer Science \& Business Media.

Porter, G. and P. Bredderman. 1997. Nonprint Formats: A Survey of the Work and Its Challenges for the Cataloger in ARL Academic Libraries. Cataloging \& Classification Quarterly 24(3-4): 125-48.

Sauperl, A. and J.D. Saye 1998. Subject Determination During the Cataloging Process: An Intensive Study of Five Catalogers. Advances in Classification Research Online 9(1): 119-38.

Silva, A.S.C., D.T. Ferreira, and L. de Azevedo Martins 2016. Development and Challenges in Old Manuscripts Cataloging: The Experience of the National Library of Portugal. Cataloging \& Classification Quarterly 54(5-6): 350- 8. https://doi.org/10.1080/01639374.2016.1199450

Svenonius, E. 2000. The Intellectual Foundation of Information Organization. Cambridge, Massachusetts: The MIT Press.

Taylor, Arlene and Joudrey, Daniel N. 2009. Subject Analysis. In The Organization of Information. Westport, Conn: Libraries Unlimited, 303-32.

Thompson, Kelly. J. 2016. More Than a Name: A Content Analysis of Name Authority Records for Authors Who Self-Identify as Trans. Library Resources \& Technical Services 60(3): 140-55. https://doi.org/10.5860/lrts.60n3.140

Wilson, Patrick. 1968. Subjects and the Sense of Position. In Two Kinds of Power: An Essay on Bibliographical Control. Berkeley: University of California Press, 69-92. 